\title{
Protocol-driven daily optimisation of venovenous extracorporeal membrane oxygenation blood flows: an alternate paradigm?
}

\author{
Kiran Shekar ${ }^{1,2,3}$, Hergen Buscher ${ }^{4,5}$, Daniel Brodie ${ }^{6,7}$ \\ ${ }^{1}$ Adult Intensive Care Services and Critical Care Research Group, the Prince Charles Hospital, Brisbane, Queensland, Australia; ${ }^{2}$ Institute of Health \\ and Biomedical Innovation, Queensland University of Technology, Brisbane, Queensland, Australia; ${ }^{3}$ Faculty of Medicine, University of Queensland, \\ Brisbane and Bond University, Gold Coast, Queensland, Australia; ${ }^{4}$ Department of Intensive Care Medicine and Centre for Applied Medical \\ Research, St. Vincent's Hospital, Sydney, NSW, Australia; ${ }^{5}$ Faculty of Medicine, University of New South Wales, Sydney, NSW, Australia; ${ }^{6}$ Columbia \\ University College of Physicians and Surgeons/New York-Presbyterian Hospital, New York, NY, USA; ${ }^{7}$ Center for Acute Respiratory Failure, New \\ York-Presbyterian Hospital, New York, NY, USA \\ Contributions: (I) Conception and design: K Shekar; (II) Administrative support: None; (III) Provision of study materials or patients: None; (IV) \\ Collection and assembly of data: None; (V) Data analysis and interpretation: None; (VI) Manuscript writing: All authors; (VII) Final approval of \\ manuscript: All authors. \\ Correspondence to: Prof. Kiran Shekar, MBBS, PhD. Adult Intensive Care Services, the Prince Charles Hospital, 627 Rode Road, Chermside, Brisbane, \\ Queensland, Australia. Email: Kiran.Shekar@health.qld.gov.au.
}

\begin{abstract}
Venovenous extracorporeal membrane oxygenation (VV ECMO) is now an established modality of support for patients with the who are failing evidence-based conventional therapies. Minimising ventilator-induced lung injury is the guiding principle behind patient management with VV ECMO. Patients with acute respiratory distress syndrome (ARDS) supported with VV ECMO are liberated from ECMO at a stage when native lungs have recovered sufficiently to support physiologic demands and the risks of iatrogenic lung injuries after discontinuation of ECMO are perceived to be small. However, native lung recovery is a dynamic process and patients rely on varying degrees of contributions from both native lungs and ECMO for gas exchange support. Patients often demonstrate near total ECMO dependence for oxygenation and decarboxylation early in the course of the illness and this may necessitate higher ECMO blood flow rates (EBFRs). Although, reliance on high EBFR for oxygenation support may remain variable over the course of ECMO, blood flow requirements typically diminish over time as native lungs start to recover. Currently, protocol-driven modulation of the EBFR based on changing physiologic needs is not common practice and consequently patients may remain on higher than physiologically necessary EBFR. This exposes the patient to potential risks because maintaining higher blood flows often requires a less restrictive fluid balance and deeper sedation. Both may be harmful in the setting of recovery from ARDS. In this article, we propose a strategy that involves daily assessments of native lung function and a protocoldriven daily optimisation of EBFR. This is followed by optimisation of sweep gas flow rate (SGFR) and the fraction of delivered oxygen in the sweep gas $\left(\mathrm{FdO}_{2}\right)$. This staged approach to weaning VV ECMO allows us to fully utilise the "decoupling" of oxygenation and decarboxylation that is possible only during extracorporeal support. This approach may benefit patients by allowing for greater fluid restriction, more aggressive fluid removal, expedited weaning of sedation and neuromuscular blocking agents (NMBAs), and early physical rehabilitation. Ultimately, prospective studies are needed to evaluate optimal VV ECMO weaning practices
\end{abstract}

Keywords: Extracorporeal membrane oxygenation (ECMO); acute respiratory distress syndrome (ARDS); awake ECMO; weaning

Submitted Mar 29, 2020. Accepted for publication Aug 05, 2020.

doi: $10.21037 /$ jtd-20-1515

View this article at: http://dx.doi.org/10.21037/jtd-20-1515

(c) Journal of Thoracic Disease. All rights reserved. 
The optimal technique for weaning venovenous extracorporeal membrane oxygenation (VV ECMO) in the setting of the acute respiratory distress syndrome (ARDS) has not been clearly established and remains variable indicating that there may be multiple techniques possible (1-3). Traditional approaches to weaning VV ECMO typically consider the ECMO blood flow rate (EBFR) as a "fixed" variable, and recommend a weaning strategy (4) based on down-titration of the sweep gas flow rate (SGFR) or the fraction of delivered oxygen in the sweep gas flow $\left(\mathrm{FdO}_{2}\right)$. While this approach is physiologically sound, it preserves the EBFR rate even though the patient's need for support may decrease as the patient approaches weaning readiness with diminishing dependency on the EBFR for adequate arterial oxygenation over time. Assuming sweep gas flow is turned on, $\mathrm{FdO}_{2}$ is set appropriately, and the gas exchange device is functioning adequately, oxygenation in the pulmonary artery during ECMO is then largely driven by the EBFR relative to the native cardiac output (CO) of the patient.

The EBFR is controlled by changing the power of the pump. It is dependent on cannula type, size and location-affecting drainage and reinfusion pressuresand on the venous return $(5,6)$. As the patient's $\mathrm{CO}$, oxygen consumption and ability to oxygenate through the native lung are dynamic variables, EBFR may be actively titrated to optimize the ECMO blood flow/CO ratio in order to achieve a desired oxygenation target $(5,7)$. Patients typically transition from a state of total or near total ECMO dependency (for oxygenation and decarboxylation) in the initial days of support to partial ECMO dependency, to some ECMO dependency, which in the majority of cases is largely for decarboxylation as ECMO weaning becomes imminent (Figure 1). In this setting, maintaining high EBFRs for the entire duration of ECMO provision may potentially lead to unnecessary and potentially harmful support levels. High EBFR relative to cannula size may cause high pressures and cellular destruction (8). In addition, the perceived need to maintain what are essentially higher than necessary EBFRs, requires the maintenance of adequate venous filling. Since negative fluid balance is one of the main treatment options in ARDS, this may impair diuresis or hemofiltration and could therefore lead to delays in weaning from VV ECMO.

This paper describes a potential alternate approach that involves daily optimisation of the EBFR and SGFR, as appropriate, leading up to weaning of SGFR and $\mathrm{FdO}_{2}$ prior to liberation from ECMO. With this in mind, there are three key steps involved (Box 1).

To allow for safe, gradual weaning of the EBFR, a daily EBFR down-titration test is performed in $300 \mathrm{~mL} / \mathrm{min}$ decrements to establish the lowest tolerable EBFR while maintaining ultra-protective ventilation and arterial oxygen saturation $\geq 88 \%$ (some centres will choose alternate oxygenation targets that, if lower, will be easier to achieve). During this early EBFR weaning phase ventilator $\mathrm{FiO}_{2}$ is maintained at $\leq 50 \%$ if tolerated, $\mathrm{FdO}_{2}$ is set at $100 \%$. If there is desaturation during the weaning process, the EBFR will be up titrated at $300 \mathrm{~mL} / \mathrm{min}$ increments to achieve target $\mathrm{SpO}_{2}$. If this is not possible to achieve, sources of continued hypoxemia should be identified, including high $\mathrm{CO}$ states, recirculation and poor gas exchange within the oxygenator. It should be noted that, similar to SGFR, EBFR can always be increased or decreased based on metabolic demands and fluctuations in CO during ongoing care between these daily tests.

Optimal haemoglobin $(\mathrm{Hb})$ targets in VV ECMO are not clearly defined. EBFR, $\mathrm{Hb}$ and CO play a critical role in ensuring optimal oxygen delivery during $\mathrm{VV}$ ECMO. Studies suggest an inverse relationship between $\mathrm{Hb}$ and EBFR in determining arterial and venous oxygen delivery, when $\mathrm{CO}$ and systemic oxygen consumption are held relatively constant (9). In this setting, although a higher $\mathrm{Hb}$ may appear justifiable, the risk-to-benefit ratio of maintaining a higher $\mathrm{Hb}$ target (e.g., $10-12 \mathrm{~g} / \mathrm{dL}$ ), is unknown. Available data indicate that a restrictive transfusion strategy is feasible on VV ECMO (10). Importantly, the chosen systemic oxygen target has a significant bearing on the EBFR required to achieve that target and aiming for higher targets than physiologically necessary may predispose a patient to the potential risks of these higher than necessary EBFRs.

Once a safe lower limit for EBFR for any given day is established, a $100 \%$ fraction of inspired oxygen test is performed by increasing ventilator $\mathrm{FiO}_{2}$ to $100 \%$ for 15 minutes while $\mathrm{FdO}_{2}$ is set at $100 \%$. $\mathrm{A} \mathrm{PaO}_{2} \geq 250 \mathrm{mmHg}$ on a positive end expiratory pressure (PEEP) of $\leq 10 \mathrm{cmH}_{2} \mathrm{O}$ is considered a successful $100 \%$ fraction of inspired oxygen test. The test can provide trends in oxygen transfer across native lungs $\left(\mathrm{VO}_{2 \mathrm{NL}}\right)$, so long as the oxygen transfer through the oxygenator $\left(\mathrm{VO}_{2 \mathrm{ML}}\right)$ remains stable. However, this trend is heavily influenced by changes in oxygen transfer, systemic oxygen consumption and CO. Interpretation of the test requires understanding the clinical context at the time it is performed.

Where available, volumetric capnometry can help 


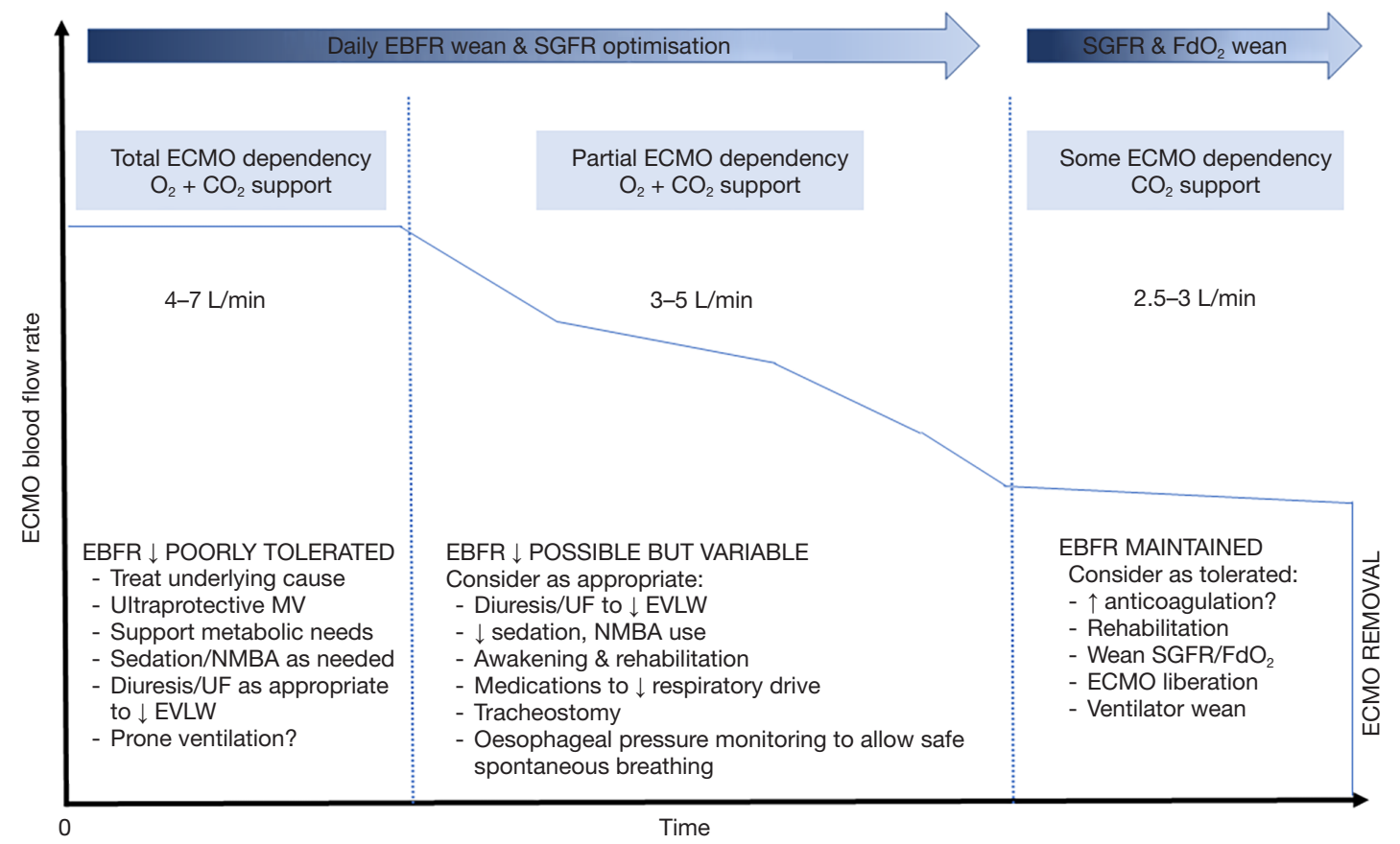

Figure 1 A typical course of VV ECMO during which a patient progresses through varying degree of ECMO dependency. There is significant variability in EBFR requirements between patients and the EBFRs chosen here are for illustrative purposes only. Patients also show great variability in their lung healing process and their physiology which is dynamic and non-linear. It may be possible to down-titrate EBFRs in most patients, which may be potentially beneficial. It is easy to up-titrate EBFRs if there is increase in the metabolic needs or the $\mathrm{CO}$ of the patient. Similarly, SGFR may need daily optimisation based on the patient's physiology and improvements in $\mathrm{CO}_{2}$ removal across the native lung over time. VV ECMO, venovenous extracorporeal membrane oxygenation; EBFR, ECMO blood flow rate; CO, cardiac output; SGFR, sweep gas flow rate; $\mathrm{CO}_{2}$, carbon dioxide; $\mathrm{FdO}_{2}$, fraction of delivered oxygen in the sweep gas flow; NMBA, neuromuscular blocking agent; UF, ultrafiltration; EVLW, extravascular lung water.

Box 1 Daily optimisation of EBFR and SGFR

(I) Protocol-driven daily optimisation of the EBFR
(II) Daily assessments of native lung function and SGFR
optimisation
(i) $100 \%$ fraction of inspired oxygen test
(ii) Physiological dead space (VD/VT)
(iii) Pulmonary system compliance and respiratory drive
(III) $\mathrm{FdO}_{2}$ and SGFR wean to liberate patient from ECMO

ECMO, extracorporeal membrane oxygenation; EBFR, ECMO blood flow rate; SGFR, sweep gas flow rate; $\mathrm{FdO}_{2}$, fraction of delivered oxygen in the sweep gas flow.

monitor the physiologic dead space $(11,12)$. Measuring carbon dioxide $\left(\mathrm{CO}_{2}\right)$ elimination across the native $\left(\mathrm{VCO}_{2 \mathrm{NL}}\right)$ and membrane $\left(\mathrm{VCO}_{2 \mathrm{ML}}\right)$ lungs can help estimate proportion of total $\mathrm{CO}_{2}\left(\mathrm{CO}_{2 \text { tot }}\right)$ eliminated through the native lungs $\left(\mathrm{VCO}_{2 \mathrm{NL}} / \mathrm{CO}_{2 \mathrm{tot}}\right)$ over time. $\mathrm{VCO}_{2 \mathrm{ML}}$ can be calculated by applying volumetric capnometry to the membrane lung and measuring $\mathrm{CO}_{2}$ at the sweep gas outlet using an infrared analyser (12). End tidal $\mathrm{CO}_{2}$ monitoring (13) may be used as a surrogate to monitor trends in $\mathrm{VCO}_{2 \mathrm{NL}}$ provided SGFR remains unchanged, when volumetric capnometry is not available. Mechanical power, plateau and driving pressures, static and dynamic compliance of the native lungs are assessed daily to ensure ongoing ultra-lung-protective ventilation. Oesophageal pressure monitoring, if available, may help measure swings in oesophageal pressure and further optimisation of transpulmonary pressures and respiratory effort.

Upon a successful $100 \%$ oxygen test, ventilator $\mathrm{FiO}_{2}$ is reduced to $\leq 50 \%$ and $\mathrm{FdO}_{2}$ and SGFR wean (step III) are commenced. Ultra-protective ventilator settings are maintained by adjusting SGFR. In the light of the recent ECMO to Rescue Lung Injury in Severe ARDS (EOLIA) 
trial (14), it may be prudent to adjust tidal volumes for a goal plateau airway pressure of $\leq 24 \mathrm{cmH}_{2} \mathrm{O}$ in conjunction with $\mathrm{PEEP} \geq 10 \mathrm{cmH}_{2} \mathrm{O}$ (corresponding to a driving pressure $\leq 14 \mathrm{cmH}_{2} \mathrm{O}$ ) and a respiratory rate of $10 \leq$ breaths per minute (15). It is important to be aware that, decreasing tidal volume $<4 \mathrm{~mL} / \mathrm{kg}$ PBW may increase atelectasis and may result in severe ventilation/perfusion mismatch and higher EBFR requirement unless PEEP is appropriately increased. Sedation and neuromuscular blocking agents (NMBAs) are adjusted according to unit policy and patient needs. In spontaneously breathing patients, SGFR is also useful to control respiratory drive and work of breathing. While maintaining protective ventilation, the intensity of mechanical ventilation is gradually increased. It is possible to measure both driving pressures during spontaneous breathing (16) to ensure protective ventilation. Oesophageal pressure monitoring, if available may be an useful tool to monitor the intensity of spontaneous effort in patients (17).

Clearly, based on CO, degree of lung rest, transpulmonary shunting/deoxygenation (18) and metabolic demands, each patient may respond differently to EBFR down-titration. As the hyperacute phase of illness starts to resolve (typically at the end of the first week, although this may vary widely, depending on the underlying pathology in any given patient), it may be possible to significantly reduce EBFRs (Figure 1) over time. This may allow for greater fluid restriction, more aggressive fluid removal, expedited weaning of sedation and NMBAs and early rehabilitation without compromising the EBFR. A welltimed tracheostomy may be a useful adjunct in this setting. Emerging data indicates that a percutaneous tracheostomy can be safely performed during ECMO (19) and an early tracheostomy may be potentially beneficial (20,21). Every patient is unique, some may be endotracheally extubated, while some others are endotracheal tube intolerant or have higher airway secretion burden or perceived work of breathing and may benefit from a tracheostomy. Ultimately, tracheostomy should be individualised to each patient's needs. The EBFR wean protocol proposed here is intended for patients with severe forms of ARDS supported with VV ECMO as a bridge to recovery. However, broadly speaking, the principles behind this strategy may also be applied to patients with end-stage lung disease who receive VV ECMO as a bridge to transplant in so far as titration of EBFR may be tailored to the needs of the patient (22), keeping in mind that the goal of ECMO support in bridge to transplant is typically to support a patient who is not able to be weaned prior to transplantation.
Once EBFRs are weaned down to the lowest possible levels while maintaining ultra-lung protection, minimal sedation and a $\mathrm{SpO}_{2} \geq 88 \%$ (typically this may occur with an EBFR of around $2.5-3 \mathrm{~L} / \mathrm{min}$ ), a typical weaning strategy targeting the incremental weaning of the SGFR or $\mathrm{FdO}_{2}$, or simply turning off the sweep gas flow to see if the patient tolerates being off support, may be attempted. Depending on the ECMO circuit used, a minimal threshold for EBFR should be defined to avoid the haemolysis that may accompany low EBFRs (23), along with thromboembolic complications. Relative increases in the intensity of anticoagulation may be indicated during the EBFR wean phase, while carefully monitoring transmembrane pressures and for haemolysis. Oxygenator performance should be monitored by measuring the post-membrane $\mathrm{PO}_{2}, \mathrm{VO}_{2 \mathrm{ML}}$, and $\mathrm{VCO}_{2 \mathrm{ML}}$ (12). It should be noted that, regardless of the EBFR, anticoagulation practices vary quite substantially around the world (24) and there is no universal agreement on the degree of anticoagulation needed for any given device or patient. There may be a higher risk of thrombotic events with the use of dual-lumen cannula (25) and a greater degree of anticoagulation may be indicated in these patients, although this remains controversial.

A variation of the technique described here has been practiced by some experienced high-volume ECMO centres (1). A technique similar to the one proposed here has been described by Grant et al. (2). Upon clinical and radiological improvement in native lung function, the authors recommend weaning EBFR to $3-4 \mathrm{~L} / \mathrm{min}$ and $\mathrm{FdO}_{2}$ to $21 \%$ prior to commencing a SGFR wean and subsequent liberation from ECMO. In contrast, we recommend daily EBFR optimisation from the day of initiating ECMO support while maintaining $\mathrm{FdO}_{2}$ at $100 \%$. Given that it may take weeks to achieve notable improvement in pulmonary compliance and radiological findings, we recommend early protocol-driven EBFR optimisation. We argue that this approach allows for more aggressive fluid restriction, which may in fact expedite improvements in pulmonary compliance and radiological findings.

Weaning EBFR through the course of ECMO is not an uncommon occurrence, yet clinicians may find it challenging to make progress when they enter a perpetual vicious circle based on the high EBFR dependency for oxygenation. Attempts at maintaining such high flow rates leads to greater volume expansion, worsening extravascular lung water, generalised fluid overload, higher sedation requirements and an inability to safely awaken the patient without compromising EBFRs. This may unnecessarily 
prolong the duration of ECMO support. The technique described here allows for a protocolized daily wean of the EBFR. Analogous to $\mathrm{FiO}_{2}$ and PEEP down-titration in the lead up to mechanical ventilation weaning, weaning EBFR may have physiologic merit. This staged approach to $\mathrm{VV}$ ECMO weaning allows us to fully utilize the "decoupling" of oxygenation and decarboxylation that is possible only during extracorporeal support.

It is important to note that, the safety margins of EBFR are not established in clinical studies (23). In future, technological refinements may allow safe EBFR wean to much lower levels (e.g., $1.5 \mathrm{~L} / \mathrm{min}$ ) than suggested in this article to predominantly support decarboxylation requirements in later stages of an ECMO run. Ideally, ECMO circuit components should be able to operate safely across a wide range of EBFRs and whether this can be achieved with current circuitry remains unclear. Although contrasting data exists on the effects of different EBFRs on haemostatic parameters in preclinical studies, which adopted similar anticoagulation regimens at both higher and lower EBFRs (26-28), clinical validation of these findings is needed. For example, even if some unfavourable haemostatic alterations were observed in pre-clinical studies with lower EBFRs, those risks may be offset by an ability to deliver evidence-based intensive care unit practices such as fluid restriction, minimization of sedation, reduced NMBA use and early rehabilitation. As our understanding of potential ECMO-induced pathophysiological injuries improves, we will be able to better titrate VV ECMO support in the future.

Approaches to weaning VV ECMO using SGFR, FdO and EBFR based approaches have risks and benefits. In an evolving field such as ECMO, all potential weaning techniques and management strategies merit robust debate. Physiology-based management approaches have not always been successful in the intensive care unit. Strategies such as the one proposed here that minimize the risks of sedation (29), prolonged NMBA use, and fluid overload $(30,31)$ as well as potentially promoting early awakening and rehabilitation (32) have been shown to be beneficial in other contexts. The challenges of performing clinical trials to meaningfully test any of the proposed weaning approaches cannot be understated but are necessary to ultimately improve patient outcomes.

\section{Conclusions}

EBFR requirements during VV ECMO are not fixed. Supporting patients with the minimum possible EBFR while on VV ECMO may have several advantages. Daily protocol-driven optimisation of EBFR during VV ECMO support may allow a more fluid restrictive and hence lungprotective strategy. The approach proposed here may facilitate early weaning of sedation, awake ECMO, early rehabilitation and possibly liberation from mechanical ventilation in selected patients.

\section{Acknowledgments}

KS acknowledges research support from the Metro North Hospital and Health service.

Funding: None.

\section{Footnote}

Conflicts of Interest: All authors have completed the ICMJE uniform disclosure form (available at http://dx. doi. org/10. 21037/jtd-20-1515). DB reports grants from ALung Technologies, personal fees from Baxter, personal fees from BREETHE, personal fees from Xenios, other from Hemovent, outside the submitted work. The other authors have no conflicts of interest to declare.

Ethical Statement: The authors are accountable for all aspects of the work in ensuring that questions related to the accuracy or integrity of any part of the work are appropriately investigated and resolved.

Open Access Statement: This is an Open Access article distributed in accordance with the Creative Commons Attribution-NonCommercial-NoDerivs 4.0 International License (CC BY-NC-ND 4.0), which permits the noncommercial replication and distribution of the article with the strict proviso that no changes or edits are made and the original work is properly cited (including links to both the formal publication through the relevant DOI and the license). See: https://creativecommons.org/licenses/by-nc-nd/4.0/.

\section{References}

1. Broman LM, Malfertheiner MV, Montisci A, et al. Weaning from veno-venous extracorporeal membrane oxygenation: how I do it. J Thorac Dis 2018;10:S692-7.

2. Grant AA, Hart VJ, Lineen EB, et al. A weaning protocol for venovenous extracorporeal membrane oxygenation with a review of the literature. Artif Organs 2018;42:605-10. 
3. Grasselli G, Mangili P, Sosio S, et al. Weaning from VV ECMO. In: Sangalli F, Patroniti N, Pesenti A. editors. ECMO-extracorporeal life support in adults. Milano: Springer Milan, 2014:317-23.

4. Vasques F, Romitti F, Gattinoni L, et al. How I wean patients from veno-venous extra-corporeal membrane oxygenation. Crit Care 2019;23:316.

5. Schmidt M, Tachon G, Devilliers C, et al. Blood oxygenation and decarboxylation determinants during venovenous ECMO for respiratory failure in adults. Intensive Care Med 2013;39:838-46.

6. Jayaraman AL, Cormican D, Shah P, et al. Cannulation strategies in adult veno-arterial and veno-venous extracorporeal membrane oxygenation: techniques, limitations, and special considerations. Ann Card Anaesth 2017;20:S11-8.

7. Brodie D, Slutsky AS, Combes A. Extracorporeal life support for adults with respiratory failure and related indications: a review. JAMA 2019;322:557-68.

8. Lehle K, Philipp A, Zeman F, et al. Technical-induced hemolysis in patients with respiratory failure supported with veno-venous ECMO - prevalence and risk factors. PLoS One 2015;10:e143527.

9. Spinelli E, Bartlett RH. Relationship between hemoglobin concentration and extracorporeal blood flow as determinants of oxygen delivery during venovenous extracorporeal membrane oxygenation: a mathematical model. ASAIO J 2014;60:688-93.

10. Agerstrand CL, Burkart KM, Abrams DC, et al. Blood conservation in extracorporeal membrane oxygenation for acute respiratory distress syndrome. Ann Thorac Surg 2015;99:590-5.

11. Raurich JM, Vilar M, Colomar A, et al. Prognostic value of the pulmonary dead-space fraction during the early and intermediate phases of acute respiratory distress syndrome. Respir Care 2010;5 5:282-7.

12. Epis F, Belliato M. Oxygenator performance and artificialnative lung interaction. J Thorac Dis 2018;10:S596-605.

13. McSwain SD, Hamel DS, Smith PB, et al. End-tidal and arterial carbon dioxide measurements correlate across all levels of physiologic dead space. Respir Care 2010;5 5:288-93.

14. Combes A, Hajage D, Capellier G, et al. Extracorporeal membrane oxygenation for severe acute respiratory distress syndrome. N Engl J Med 2018;378:1965-75.

15. Abrams D, Schmidt M, Pham T, et al. Mechanical ventilation for acute respiratory distress syndrome during extracorporeal life support. Research and practice. Am J
Respir Crit Care Med 2020;201:514-25.

16. Bellani G, Grassi A, Sosio S, et al. Plateau and driving pressure in the presence of spontaneous breathing. Intensive Care Med 2019;45:97-8.

17. Yoshida T, Brochard L. Esophageal pressure monitoring: why, when and how? Curr Opin Crit Care 2018;24:216-22.

18. Gattinoni L, Vassalli F, Romitti F, et al. Extracorporeal gas exchange: when to start and how to end? Crit Care 2019;23:203.

19. Salna M, Tipograf $Y$, Liou $P$, et al. Tracheostomy is safe during extracorporeal membrane oxygenation support. ASAIO J 2020;66:652-6.

20. Grewal J, Sutt AL, Cornmell G, et al. Safety and putative benefits of tracheostomy tube placement in patients on extracorporeal membrane oxygenation: a single-center experience. J Intensive Care Med 2020;35:1153-61.

21. DiChiacchio L, Boulos FM, Brigante F, et al. Early tracheostomy after initiation of venovenous extracorporeal membrane oxygenation is associated with decreased duration of extracorporeal membrane oxygenation support. Perfusion 2020;35:509-14.

22. Tipograf Y, Salna M, Minko E, et al. Outcomes of extracorporeal membrane oxygenation as a bridge to lung transplantation. Ann Thorac Surg 2019;107:1456-63.

23. Gross-Hardt S, Hesselmann F, Arens J, et al. Low-flow assessment of current ECMO/ECCO2R rotary blood pumps and the potential effect on hemocompatibility. Crit Care 2019;23:348.

24. Protti A, Iapichino GE, Di Nardo M, et al. Anticoagulation management and antithrombin supplementation practice during veno-venous extracorporeal membrane oxygenation: a worldwide survey. Anesthesiology 2020;132:562-70.

25. Shafii AE, Brown CR, Murthy SC, et al. High incidence of upper-extremity deep vein thrombosis with dual-lumen venovenous extracorporeal membrane oxygenation. J Thorac Cardiovasc Surg 2012;144:988-9.

26. Ki KK, Passmore MR, Chan $\mathrm{CHH}$, et al. Effect of ex vivo extracorporeal membrane oxygenation flow dynamics on immune response. Perfusion 2019;34:5-14.

27. Fuchs G, Berg N, Broman LM, et al. Flow-induced platelet activation in components of the extracorporeal membrane oxygenation circuit. Sci Rep 2018;8:13985.

28. Ki KK, Passmore MR, Chan CHH, et al. Low flow rate alters haemostatic parameters in an ex-vivo extracorporeal membrane oxygenation circuit. Intensive Care Med Exp 2019;7:51. 
29. Shekar K, Grewal J, Lisa Sutt A, et al. Individualizing sedation in acute respiratory distress syndrome patients on extracorporeal membrane oxygenation. ASAIO J 2019;65:e44-5.

30. McCanny P, Smith MW, O'Brien SG, et al. Fluid balance and recovery of native lung function in adult patients supported by venovenous extracorporeal membrane oxygenation and continuous renal replacement therapy. ASAIO J 2019;65:614-9.

Cite this article as: Shekar K, Buscher H, Brodie D. Protocoldriven daily optimisation of venovenous extracorporeal membrane oxygenation blood flows: an alternate paradigm? J Thorac Dis 2020;12(11):6854-6860. doi: 10.21037/jtd-20-1515
31. Schmidt M, Bailey M, Kelly J, et al. Impact of fluid balance on outcome of adult patients treated with extracorporeal membrane oxygenation. Intensive Care Med 2014;40:1256-66.

32. Abrams D, Javidfar J, Farrand E, et al. Early mobilization of patients receiving extracorporeal membrane oxygenation: a retrospective cohort study. Crit Care 2014;18:R38. 